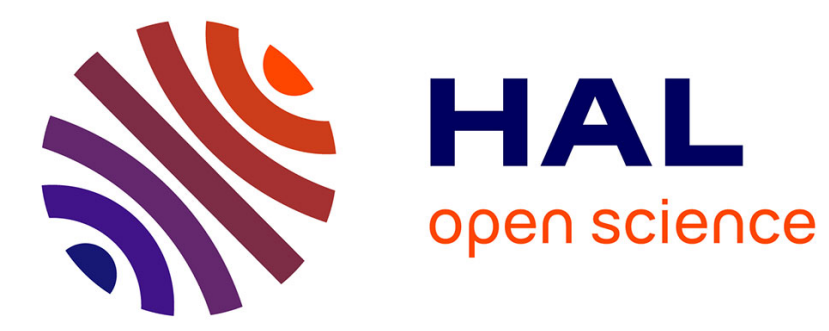

\title{
Electrokinetics: insights from simulation on the microscopic scale
}

Benjamin Rotenberg, Ignacio Pagonabarraga

\section{To cite this version:}

Benjamin Rotenberg, Ignacio Pagonabarraga. Electrokinetics: insights from simulation on the microscopic scale. Molecular Physics, 2013, 111 (7), pp.827 - 842. 10.1080/00268976.2013.791731. hal-01916530

\section{HAL Id: hal-01916530 \\ https://hal.sorbonne-universite.fr/hal-01916530}

Submitted on 8 Nov 2018

HAL is a multi-disciplinary open access archive for the deposit and dissemination of scientific research documents, whether they are published or not. The documents may come from teaching and research institutions in France or abroad, or from public or private research centers.
L'archive ouverte pluridisciplinaire HAL, est destinée au dépôt et à la diffusion de documents scientifiques de niveau recherche, publiés ou non, émanant des établissements d'enseignement et de recherche français ou étrangers, des laboratoires publics ou privés. 


\title{
TOPICAL REVIEW
}

\section{Electrokinetics: insights from simulation on the microscopic scale}

\author{
Benjamin Rotenberg $^{a *}$ and Ignacio Pagonabarraga ${ }^{b}$ \\ ${ }^{a}$ CNRS, UPMC Univ Paris 06, ESPCI, UMR 7195, PECSA, F-75005, Paris, France; \\ ${ }^{b}$ Departament de Fúsica Fonamental, Universitat de Barcelona, 08028-Barcelona, Spain
}

(March 22, 2013)

\begin{abstract}
Electrokinetic effects, i.e. the coupled hydrodynamic and electric phenomena which occur near charged interfaces, constitute a challenge to theorists due to the variety of length and time scales involved. We discuss recent advances in the modelling of these phenomena, emphasizing the interplay between the molecular specificity and the collective induced flows that emerge. We discuss the complementary simulation methodologies that have been developed either to focus on the molecular aspects of electrokinetics or on their effective properties on larger scales, as well as the proposed hybrid schemes that can incorporate both aspects. We highlight the insights that molecular studies have brought on the nature of interfacial charges and their implications for kinetic phenomena in confined fluids and also discuss advances in a number of relevant contexts.
\end{abstract}

\section{Introduction}

Electrokinetic effects are the coupled hydrodynamic and electrostatic phenomena occurring at a charged solid/liquid or liquid/liquid interface. Such interfaces are ubiquitous in nature and in technology. For instance, the surface of many minerals (e.g. silica or clays) and most biological molecules (e.g. DNA and proteins) are charged. The charge on the surface of colloids, membranes and microfluidic devices also plays an important role for their applications. The origin of electrokinetic couplings lies in the fact that the fluid is charged in the vicinity of the surface. Indeed, the competition between the electrostatic attraction to the surface of the counterions compensating its charge and the entropic gain for their dissolution in the fluid induces a local violation of electroneutrality. The resulting charged region, the "double-layer", extends over the so-called Debye screening length, which is typically in the nanometer range.

In the simple case of electro-osmosis near a fixed charged wall, an electric field accelerates the fluid in the double-layer region where the fluid is charged. Momentum is then slowly transferred to the rest of the fluid by viscous stresses even in the regions where it is electrically neutral. This is can be exploited e.g. for pumping in micro- and nanofluidic devices [1,2]. The feedback between the stresses that local charges induce in the solvent and the distortion in the ionic distribution due to advection by the solvent are at the origin of the complexity of electrokinetic phenomena. The interplay between the different scales gives rise to a rich phenomenology. The interactions between the colloidal particles can be modified by the dynamic polarization of the ionic cloud under an electric field, resulting in a complex phase diagram as a function of the field frequency, its amplitude and the

*Corresponding author. Email: benjamin.rotenberg@upmc.fr 
colloid concentration [3, 4]. Recent experiments have also demonstrated the rich electrokinetic behaviour of superhydrophobic surfaces [5]. New concepts for controlling electro-osmotic flows (EOF) in microfluidic devices have been introduced, such as using a self-assembled monolayer as the insulator of a flow field-effect transistor [6].

Theoretical advances in recent years have addressed the relevance of the doublelayer extent when it competes with other characteristic length scales, such as a macromolecule size, or the pore size in the case of electrolytes confined in porous media [7]. Although the standard theoretical treatment, built on the PoissonNernst-Planck equation, is a mean field description of the diffusion of ions that can account for short range ionic interactions, only recently have theoretical studies started to analyze the impact of the ionic size on the electrokinetic response of an electrolyte both in the linear [8] and nonlinear regimes [9]. For multivalent ions, it is also necessary to understand the relevance of electrostatic correlations and their impact both in the stability of a homogeneous electrolyte solution and on the effective interactions on larger scales [10]. In this review we will focus on the advances and understanding gained through computer simulations; the latter in fact provide a means to validate a number of assumptions and predictions made by these recent theoretical advances.

From the modelling perspective, the challenge lies in the range of length and time scales involved, from the molecular scale which governs the interactions between the solid and the fluid to the macroscopic flows which arise from the long-ranged hydrodynamic and electrostatic couplings. A number of complementary strategies have been developed accordingly, from molecular simulation to analytical theories based on a continuous description of the fluid. A few years ago, we discussed a number of them, focussing on the mesoscopic simulation techniques [11]. In the present review, we first consider the recent advances in such approaches, which vary in their explicit/implicit treatment of the solvent and microions. In particular, we emphasize the recent progress achieved with simulation schemes based on kinetic theory. We then focus our discussion on the many insights on electrokinetic effects gained from molecular simulations. The latter have been extremely useful in this field over the past decade and the community has been very active in the last few years. We finally illustrate recent applications of these methods in various contexts, including "traditional" fields (colloids and polyelectrolytes) for which new methodological and numerical developments have allowed significant progress, and hitherto unexplored ones (e.g. ionic liquids) for which molecular simulations provided the first look into new microscopic mechanisms.

\section{Simulation strategies}

\subsection{Need for a multiscale approach}

The study of electrokinetics involves the understanding of the common motion of solvent and dissolved ions on consistent grounds. The motion of an electrolyte in a confined medium or of a suspension of charged colloids or polyelectrolytes involves a diversity of length (and the corresponding time) scales that have to be captured correctly. This requires to describe the motion of dissolved ions and their interaction with the solvent, their attraction to charged surfaces and the motion of larger solutes. Colloids or polymers, with sizes between a few nanometers and microns, build a diffuse layer of ions around them. Indeed, while global electroneutrality is always preserved, local electroneutrality is broken due to the competition between electrostatic attraction and entropic gain for the ions to dissolve in the solvent. This 
induces an inhomogeneous distribution of both counter- and coions around large suspended solutes. Salt then also plays an important role, because it determines the extent of this double-layer.

For usual salt concentrations, the double-layer extends over a few nanometers. Hence, there may be an order of magnitude difference between the typical ion and solvent sizes and the length scales over which local charge reorganizes in the fluid. An additional order of magnitude difference may exist between the ionic density inhomogeneities and the macromolecular sizes. In turn, the motion of large solutes modifies the flow field around them and this flow drags ions, thereby distorting their equilibrium distribution, i.e. inducing polarization. An analogous situation occurs for electrokinetics in a porous medium. In this case the solid porous medium does not displace but perturbs the fluid flow in the pores and the pore size plays the role of the macromolecular size. It is obviously not possible to carry out firstprinciples atomistic simulations for such heterogeneous and structured systems out of equilibrium, but one can take advantage of the above-mentioned separation of length scales to develop rather efficient mesoscopic approaches depending of the level of description that needs to be addressed. Table 1 summarizes the various methods discussed in the present review.

Table 1. Simulation strategies discussed in the text, depending on how they account of the solvent and the microions (see text for abbreviations).

\begin{tabular}{ccc}
\hline & Explicit Solvent & Implicit Solvent \\
\hline Explicit Ions & MD & BD with HI \\
& LB + MD, RET & RET \\
\hline Implicit Ions & DPD, LA or SPH & BD with PMF and HI \\
& Hybrid LB & or Green Function \\
\hline
\end{tabular}

Atomistic simulations are routinely used to assess the detailed structure of ions on solid surfaces. Effects such as the relevance of surface heterogeneity, ionic correlations or ionic specificity can only be addressed by simulating individual ions and accounting for their relative affinity for water, and for how they distort the local structure of the solvent around them. This requires the use of reliable force fields. In this respect, the use of $a b$ initio techniques has been very helpful to understand the emergence of interactions of ions in water and the relevance of hydration on the effective structures that dissolved ions can display. Due to their computational cost, such approaches generally focus on the equilibrium behaviour of a small number of ions, usually in the bulk - although recently some studies also investigated ions at the water-air interface with $a b$ initio simulations. From the perspective of electrokinetic phenomena, such simulations allowed to propose force fields and understand the power and limitations of existing potentials. Methodological aspects of atomistic simulations will be presented in section 2.4 while insights gained from molecular simulations will be presented in detail in section 3 . We first discuss mesoscopic simulations.

\subsection{Mesoscopic simulations}

Given the computational cost of fully atomistic simulations, a number of effective, coarse-grained strategies have been developed to reach larger length and longer time scales, characteristic of many of these heterogeneous systems where electrokinetic effects are relevant [11]. As most space is occupied by the solvent, describing it the at a coarse-grained level constitutes the first, obvious approach to save computing time and increase the scales that can be covered numerically. Such approaches consider that the molecular details of the solvent are not relevant and regard it 
either as a continuous fluid medium or as an underlying, fixed structureless frictional medium. Even at this implicit level, the solvent dynamics can significantly affect the ion dynamics and modify the electrokinetics of charged macromolecules or porous media. However, accounting for the collective dynamics of the solvent is not obvious, since it has to be carried out consistently with the ionic motion.

The simplest approach considers that the solvent is at rest and is not affected by the moving dissolved ions; the latter, of size $a$, experience an effective friction force proportional to their velocity $\mathbf{v}$ and related to the solvent viscosity $\eta$ by Stokes' law, $\mathbf{F}=-6 \pi \eta a \mathbf{v}$. An additional random force, ensuring detailed balance, is also included to reach proper thermal equilibrium. The electrostatic properties of the solvent enter only through its effective dielectric constant $\epsilon_{r}$ corresponding to the Primitive Model. For large friction coefficients the velocity relaxes instantaneously and solving the ion dynamics together with their electrostatic interactions reduces to a Brownian Dynamics (BD) description of charged ions. Such an approach accounts for electrostatic correlations and captures molecular specificity associated to the dissolved ions and the molecular details of the solvent enter only through the effective force between ions. In the presence of bounding walls, or in the study of the electrokinetics of macromolecular suspensions, it is also possible to introduce specific interactions between the ions and different constraining geometries to account for the differential affinity of ionic species to the heterogeneous environment they experience (see section 3).

This approach, however, disregards the fact that the solvent evolves together with the dissolved ions. The local fluid flow displaces and distorts the ion distribution and in turn, local charge accumulation of the dissolved ions accelerates the solvent. These dynamic couplings and their corresponding feedbacks do not allow for generic analytic solutions and already pose a number of numerical challenges. The dynamic coupling between the solvent and the moving ions can be taken into account by including the friction felt by the ions due to the solvent motion induced by the rest of the suspended solutes. A forced point particle induces a flow around it that can be described using the Oseen tensor. The expression for the flow field can be corrected to account for the size of the ions, leading to a configuration-dependent friction which follows the form derived by Rotne and Prager [12]. This approach, that accounts for the motion of the solvent as a continuum and leads to effective manybody interactions among ions, has been generalized to account for the effect of a confining solid boundary. The long-range nature of these hydrodynamic interactions leads to poor scaling with system size, and these methods are restricted to unbound systems or to simple geometric constraints. Complementary approaches that deal with hydrodynamic interactions under generic confinement have been developed, which exploit ideas analogous to the Ewald summation or particle-particle particlemesh techniques in electrostatics [13].

An alternative strategy is to account for an explicit, coarse-grained solvent. This is computationally more intensive because the solvent has to be tracked, but it avoids the poor scaling of the implicit methods because all dynamic rules are local. Such strategies account for effective, soft interactions among solvent particles that ensure proper thermodynamic equilibrium and recover the hydrodynamic, collective behaviour of the solvent, although without resolving its detailed local structure. For example, Dissipative Particle Dynamics (DPD) models the solvent as point particles that interact through soft conservative forces and adds a local thermostat that conserves momentum $[14,15]$. The Lowe-Anderson (LA) thermostat also provides a means to model coarse-grained solvents with correct hydrodynamics [16] and Smoothed Particle Hydrodynamics (SPH) consistently recovers hydrodynamics from an effective local dynamics of the solvent [17]. For DPD, Groot proposed an 
extension to deal with electrolytes [18]. To avoid the collapse of oppositely charged point ions, local charges are smeared out around each DPD particle. This method then provides a natural coupling between electrostatics and fluid flow, although smearing the charge makes it hard to keep an appropriate separation of length scales if the method should be applied on large scales. Combining a DPD solvent with fully resolved ions has also been exploited to study a variety of electrokinetic processes by restricting the achievable system sizes to the nanometer range [19].

To reach longer scales, one can not only coarse-grain the solvent but also average out the degrees of freedom associated to the microions. Since their dynamics is usually much faster than that of macromolecules, one can include the effect of the former into a potential of mean force between the macroions. In equilibrium the standard example of such an approach is the well-known DLVO theory [20]. Although the DLVO potentials have been extensively used in Brownian dynamics simulations, where the solvent, also implicit, is regarded as a passive frictional medium, much less has been done to include in a consistent way the effect of ions on macroions with a hydrodynamic description for the implicit solvent [21]. At the linearized level, within the Debye-Hückel regime, Ajdari and Long derived the electrokinetic Green function of an electrolyte in response to applied electric fields [22]. It is then possible to carry out Brownian dynamics simulations of charged macroions with an implicit solvent and without having to deal explicitly with the dynamics of salt and counterions, as exploited by Löwen and coworkers to study the long-time dynamics of charged colloidal suspensions [23].

\subsection{Kinetic approaches}

An alternative approach to particle-based methods, as described in the previous section, is to develop kinetic models that capture the relevant degrees of freedom and which are still formulated through local rules, hence ensuring good scalability. A powerful technique to simulate hydrodynamics is the lattice-Boltzmann method (LB). In LB one evolves dynamically the one-particle density distribution of solvent molecules $f(\mathbf{r}, \mathbf{v}, t)$ on a lattice where both time and space are discretized [24, 25]. At each time step, $f$ relaxes toward a local equilibrium distribution, which amounts to a low-velocity expansion of the Maxwell-Boltzmann distribution, and is also propagated to the local neighboring nodes according to the corresponding velocity $\mathbf{v}$. The relaxation rate determines the solvent viscosity and the amplitudes that characterize the local equilibrium distribution set the speed of sound. The equilibrium properties of the solvent are only determined by its compressibility. It has been shown that LB recovers the hydrodynamic behaviour on long length and time scales. LB combines local rules with a lattice description which makes the approach very efficient numerically. Moreover, the purely local dynamics makes this approach very flexible to model complex fluids in arbitrary geometries [26, 27].

Since LB is based on the Boltzmann equation, it offers a solid theoretical basis to address the dynamics of generic fluids. For example, in kinetic theory the impact of the finite size of the solvent in the collision kernel was accounted for by Enskog and later generalized into the Revised Enskog Theory (RET). This idea has been exploited to generalize the LB approach and propose a lattice model that includes, effectively, the finite size of the ions [28]. The corresponding kinetic model keeps most of the advantages of LB and includes the resolution of the solvent size. This approach works naturally on smaller scales, becoming therefore more intensive computationally, but offers the possibility to assess the relevance of solvent granularity on the dynamics of fluids on small scales.

These complementary kinetic approaches have been used as a basis to address 
electrokinetics. To this end, one has to couple the dynamics of dissolved ions in such an effective treatment for the solvent dynamics. It is possible to carry out such a program both with explicit or implicit ions. The latter case, which allows to reach larger time and length scales, describes the ions in terms of their local densities. One possibility is then to setup local dynamic rules that recover the advection-diffusion motion of ionic densities [29-31]. As a result, charged densities are advected by the local flow, but local charge buildup also acts as an effective force accelerating the solvent distribution function $f$. The Poisson equation needs to be solved at each time step to determine the local electric field acting both on the solvent and the ionic densities. The long range nature of the electrostatic forces requires that local charge conservation is ensured also at the solid/fluid boundaries; this requires a careful implementation of the local updating rules for the charged species. The performance of the complementary kinetic approach of LB and the particle-based DPD to simulate electro-osmotic flows has been analyzed, and the relative strengths of each strategy have been discussed in this context [32].

This approach has been used to study the sedimentation of charged colloids of different sizes as well as the dynamics of electrolytes in porous media. The methods builds on a free energy functional model for the charged species. Hence, it can be generalized to simulate at a coarse-grained level the electrokinetics of non-ideal fluids and treat ions at liquid-liquid interfaces [11]. The approach needs to resolve the charge distribution of the ions on the lattice, which sets the natural length scale that can be resolved in simulations. The Lattice RET has also been extended to account for electrolytes. In this case the charged species are treated at a more mesoscopic level and enter as a distribution function [33, 34]. The method is then analogous to that of a multi-component mixture where the corresponding collision kernels have to be introduced for the different species and it is through these terms that ion specificity can be accounted for. This kinetic model treats solvent and electrolytes consistently because it resolves to the same degree of accuracy the molecular details of solvent and ions, at the level of their mean densities, thereby providing a more efficient scheme than MD. This approach resolves the ion structure and hence works naturally at smaller scales than the former approach. The computational costs of solving the Poisson equation in these lattice models has also led to new proposals of hybrid schemes to improve their performance [35].

An alternative method, closer in spirit to the Primitive Model, considers a LB approach for the solvent but resolves individual ions. The latter are treated as in a standard MD scheme with the addition of a local friction force if the ions do not move at the same velocity as the local solvent. Momentum conservation ensures that this force also affects the fluid, enforcing the appropriate feedback between fluid flow and ionic motion [35]. Individual ionic resolution is not consistent with the continuous description of the solvent and reduces the typical length scales that can be covered. Nevertheless, this approach has been successfully used to study the electrokinetics of nanocolloids and polyelectrolytes. The use of a kinetic approach also offers more flexibility for the understanding of the kinetics of charged solutes. For example, in LB the distribution function $f$ has also a probabilistic interpretation associated with the probability that a given density moves or stays at a given node. This probability can be used, in the case of tracers, to compute not only the average behaviour but also correlation functions. Moment Propagation [36], which exploits this idea, has been used to analyze the velocity autocorrelation functions and diffusion coefficients of charged tracers in porous media to understand the relative diffusivity and how the attraction to the solid walls affects its behaviour [37].

The same kinetic approach has also ben used to model, on larger scales, the motion of ions by a density distribution diffusing in a friction, passive medium. 
Exploiting the analogy between the Nernst-Planck and the Fokker-Planck equations, one can develop a lattice method for the phase space distribution, $f_{k}(\mathbf{r}, v, t)$, for ions on species $k$ that conserves particle number and includes the electrostatic interactions, again by solving the corresponding Poisson equation. Lattice methods, which are quite efficient, can then be used to simulate the dynamics of charged ions at large length and time scales, at the expense of neglecting ionic dynamic correlations $[38,39]$.

\subsection{Microscopic scale: molecular dynamics}

All-atom molecular dynamics simulations (MD) allow to investigate on the microscopic scale the interfacial phenomena that govern electrokinetic effects on larger length and longer time scales. The trajectory of each atom is determined by numerically solving Newton's law of motion in which forces are modelled using a classical force field (the computational cost of first-principles simulations has to date not allowed to reach the relevant length and time scales for electrokinetic phenomena). In the present context, relevant information can be obtained both from the analysis of equilibrium trajectories (mainly in the framework of Green-Kubo theory) and from the response to an external perturbation with Non-Equilibrium Molecular Dynamics (NEMD). As discussed below, the Green-Kubo strategy has been introduced for hydrodynamic flows by Bocquet and Barrat [40,41] and for electro-osmotic flows by Marry et al. [42] and Dufrêche et al. [43]. NEMD can be implemented by applying a force to determine hydrodynamic properties and discuss the implications for electrokinetic flows, or by applying an electric electric field. Early works following the latter strategy for the study of EOF in nano-channels include that of Freund [44] and Qiao and Aluru [45], while the former was followed e.g. recently by Wang et al. [46]. Finally, in some cases both approaches have been compared on the same system, concluding to a good agreement [47, 48]. Figure 1 illustrates an application of NEMD to determine velocity profiles under the effect of a pressure gradient, for an aqueous solution confined in a $4.5 \mathrm{~nm}$ wide slit pore, whose charged surface consists of montmorillonite clay [49].

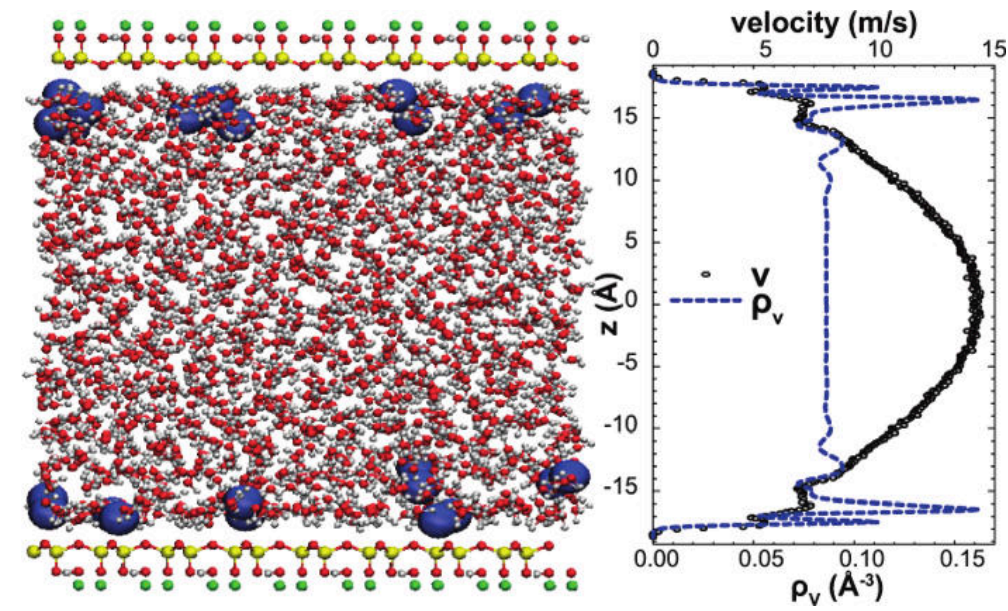

Figure 1. Non-equilibrium molecular dynamics (NEMD) simulations allow the determination of velocity profiles under the effect of external forces, taking into account the effect of molecular details. In this particular case, a uniform body force mimicking an applied pressure gradient is applied to an aqueous solution confined in a $4.5 \mathrm{~nm}$ wide slit pore, whose charged surface consists of montmorillonite clay [49]. The analysis of atomic trajectories then provides the velocity profile and allows to assess the validity of continuous theories. The layering of water molecules (oscillations in the density $\rho_{V}$, right) results in oscillations of the velocity profile $v$ near the surface. Reprinted with permission from Botan et al., J. Phys. Chem. C, 115, 16109 (2011). Copyright (2011) American Chemical Society. 
Although this is not the purpose of the present review, it is worth underlining a few general features of molecular simulations [50]. The choice of the force field is obviously an important issue if one wants to realistically represent a given system. However valuable insights can be obtained from simple models if one is only interested in generic trends, e.g. by systematically tuning the attraction between a Lennard-Jones fluid and the wall to cover a range of "hydrophilic" to "hydrophobic" surfaces. The long-range nature of electrostatic interactions requires a proper treatment of the interactions with all charged image particles. Ewald sums or particle mesh constitute standard techniques that account for the impact of image charges on the behaviour of the particles and introduce additional computational costs [51]. Similarly, the long range of hydrodynamic couplings would in principle require a systematic size-scaling analysis. It is know, for example, that hydrodynamic interactions between periodic images reduce the diffusion coefficient in a finite simulation box $[52,53]$. Extrapolation to inifite box size results for the popular SPC/E water model in a diffusion coefficient of $\sim 3.010^{-9} \mathrm{~m}^{2} \cdot \mathrm{s}^{-1}$ [54], much larger than the experimental value $\left(\sim 2.310^{-9} \mathrm{~m}^{2} . \mathrm{s}^{-1}\right)$ which is close to the one obtained with typical box sizes. Nevertheless, not all properties are similarly affected and the presence of walls also modifies the hydrodynamic couplings [41].

The next section discusses general insights relevant to electrokinetic phenomena gained from molecular dynamics simulations, while the following one focusses on a few applications of simulations on the microscopic scale (not exclusively all-atom simulations).

\section{Insights from simulation on the microscopic scale}

Molecular simulations have been extensively used in the last two decades to investigate confined fluids and hydrodynamics under extreme confinement, from nanopores of tens of molecular diameters down to nanochannels with radii of a single molecule. An example of such situation can be found with water in carbon nanotubes [55]. Reviews of the simulation of hydrodynamic flows under confinement can be found elsewhere (see e.g. $[1,56]$ ) and we focus here on studies relevant for electrokinetic effects, more precisely pertaining to the origin of the surface charge, interfacial properties of water, hydrodynamics of confined fluid and wettability, and the effect of ions on the flow.

\subsection{Origin of the surface charge}

Electrokinetic effects occur at an interface due to the presence of charge, which may arise from various mechanisms. Microscopic simulations have contributed to the better understanding of the possible origins of this interfacial charge.

Dissociation of surface groups A very common source of surface charge is the dissociation of surface groups, often as the result of an acid-base reaction. For example, the surface of oxides may display hydroxyl (X-OH) groups which can react with water or other Brönsted bases into negatively charged $\mathrm{X}-\mathrm{O}^{-}$sites and an aqueous proton, or oxygen atoms with can react with water or other Brönsted acids to form a positively charged protonated group. The resulting charge of the surface thus depends on the $\mathrm{pH}$ of the solution and the acidity constants of the various surface sites. The latter are not always easy to determine experimentally, especially on surfaces which display different acido-basic sites, because titration curves only reflect the overall behaviour. Site-specific information can be obtained only indirectly, by fitting the various acidity constants to reproduce the experimental data, 
a procedure which is in general non-univocal.

Several strategies have been proposed to determine the acidity of surface sites from ab-initio simulations. For example, Leung et al. investigated the acid-base behaviour of the water-silica interface by introducing a reaction coordinate for the deprotonation of surface groups [57]. Sprik and collaborators have developed a methodology allowing to determine acidity constants from density functional theory (DFT) based molecular dynamics simulations. This method, which extends Marcus theory for redox reactions to proton transfer, consists in computing the deprotonation free energy by thermodynamic integration. It was originally introduced for solutes [58] but later extended to surface sites of solids. For example, this method has been applied to the important cases of quartz and alumina surfaces, where the acidity of silanol $(\mathrm{Si}-\mathrm{OH})$ and aluminol $(\mathrm{Al}-\mathrm{OH})$ sites could be evaluated [59]. It was even possible to differentiate the various silanol groups on the quartz surface and it was found that "out-of-plane" silanols have a strong acidic character $\left(\mathrm{p} K_{a}=5.6\right)$ while "in-plane" silanols are slightly basic $\left(\mathrm{p} K_{a}=8.5\right)$, as a result of different hydrogen bond patterns with interfacial water molecules [60]. More recently, the same methodology was used by Tazi et al. to analyze the acidity of chemically different sites $\left(\mathrm{Si}-\mathrm{OH}, \mathrm{Al}-\mathrm{OH}\right.$ and $\left.\mathrm{Al}-\mathrm{OH}_{2}\right)$ on the edge of clay minerals [61].

Ions at an interface A key issue for electrokinetic effect is the ionic charge distribution at the interface. While the mesoscale electrokinetics of the diffuse layer can be well understood in the framework of continuum approaches, molecular simulations played a pivotal role in improving the knowledge of ions in the first molecular layers on the surface. There is a large body of molecular simulation studies of ions at interfaces and several reviews are available [62], in particular to explain ion specificity at the air-water interface [63] or at the surface of proteins [64]. One can for example mention the study of ion specific adsorption at hydrophobic solid surfaces [65], which plays an important role in electrokinetic effects, as will be discussed below, or the effect of ionic correlations with highly charged surfaces and/or mutlivalent ions [66]. An interesting aspect of ions at interfaces, whose importance has been discovered only recently, is the role of interfacial fluctuations [67, 68]. The consequences of the latter on electrokinetic effects have to date not been considered.

Neat water-air interface The fact the air bubbles present a finite electro-phoretic mobility [69] and the finite electric potential difference across the water-air interface have raised the question of the possible presence of charge at the interface between liquid water and its vapour. Classical and ab-initio molecular dynamics simulations allowed to investigate several hypotheses put forward by experimentalists and to propose new interpretations of these observations. Even in the absence of dissolved ions, water auto-ionization results in the production of hydroxide $\left(\mathrm{OH}^{-}\right)$ and hydronium $\left(\mathrm{H}_{3} \mathrm{O}^{+}\right)$ions. It has been suggested that the differential adsorption of hydronium and hydroxide ions at the water-vapour interface, may result in an overall negative charge of the interface. Vácha et al. investigated the adsorption of both ions at the water-alkane, water-vapour and water-rigid walls interfaces using MD simulations [70]. They found that all these surfaces exhibit a strong affinity for hydronium ions but no siginificant accumulation of hydroxide. Combining photoelectron spectroscopy and MD simulations with polarizable potentials, Winter et al. provided further evidence for the lack of appreciable surface hydroxide ion adsorption [71]. The polarization of the water/vapour can in fact be explained without invoking the adsorption of ions, due to the asymmetry of the hydrogen bond pattern and to charge transfer at the interface. The former aspect was for example studied by Kühne et al. using larg-scale ab-initio simulations [72], who found in agreement with sum-frequency generation spectroscopy no evidence supporting a 
large number of hydrogen bond acceptor-only species at the interface. The latter aspect, namely charge redistribution/transfer has been found to play an important role in two recent ab-initio simulations studies $[73,74]$.

\subsection{Interfacial properties of water}

Dielectric tensor The dielectric response of water near an interface is no longer isotropic and depends both on the distance to the surface and the nature of the latter, as illustrated in Figure 2. Bonthuis et al. analyzed the profiles of the static permittivity tensor of water at hydrophilic and hydrophobic interfaces [75, 76]. Such an anisotropy also arises around solutes [77]. They first found that the response was linear up to high external electric field strengths (which may occur at charged interfaces). Then, the dipole contribution dominates the dielectric response parallel to the interface, but higher order multipoles cannot be neglected to describe the perpendicular one. Introducing the space-dependent permittivity tensor in a mean-field theory improves the predictions for the ion distribution at a single charged interface and the interfacial capacitance. Finally, the difference in the interfacial dielectric response between hydrophilic and hydrophobic surfaces can be quantified by the concept of dielectric dividing interface, defined by analogy with the Gibbs dividing interface, which provides a rather simple way to introduce these interfacial effects in a modified Poisson-Boltzmann description. Combining the effects of dielectric and viscosity profiles in this continuum approach, Bonthuis and Netz recently analyzed the effect of these properties of interfacial water on the surface capacitance, the electro-osmotic mobility and the electric surface conductivity [78].

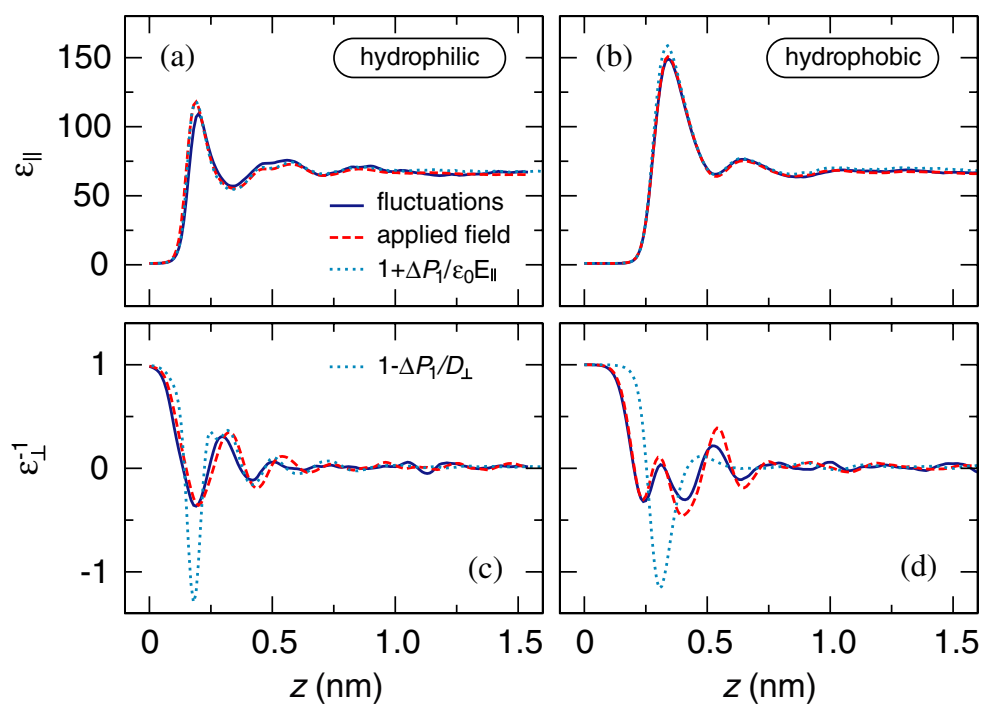

Figure 2. Parallel and inverse perpendicular dielectric functions for water near hydrophilic and hydrophobic surfaces [75]. Reprinted with permission from Bonthuis et al., Phys. Rev. Lett., 107, 166102 (2011). Copyright (2011) by the American Physical Society.

Density fluctuations The presence of an interface also imposes constraints on the fluctuations of the water density. This idea, introduced by Chandler two decades ago [79], is in fact not restricted to water, but its application to specific surfaces to quantify their hydrophobicity required the use of molecular simulations. While near a hydrophilic surface they follow the same Gaussian statistics as in the bulk, near a hydrophobic surface the probability of large fluctuations resulting in a water 
depletion is much larger [80]. The associated free energy cost can be related to the contact angle of water on the surface and is a much more reliable measure of local hydrophobicity than the water density profile [81]. Another interesting insight made possible by MD simulation is the accurate description of the water surface with the concept of instantaneous interface [82], which revealed that the water-air interface is in fact layered, even though the mean density profile in the laboratory frame appears very smooth. The implications of these concepts (density fluctuations and instantaneous interface) for hydrodynamic properties have apparently not yet been considered.

Dynamic properties From the dynamic point of view, the presence of an interface also hinders the reorientation of water molecules. The main mechanism for water reorientation consists in large-amplitude jumps between a hydrogen-bond partner to another one [83-85]. Near a surface (or around solutes), the smaller number of available molecules for such a jump to occur leads to a slowing down of the reorientation $[86,87]$. However the hydrogen bond strength also comes into play so that the reorientation dynamics depends non-monotonically on the surface hydrophilicity [88]. These microscopic mechanisms might partly explain why the dynamics of water near surfaces can be captured in simple free-energy based models only at the price of introducing spatially dependent diffusion coefficient [89]. Water in the solvation shell of ions is also affected by their electric field [90, 91]. All these features contribute to the overall interaction of the fluid with the surface, hence in principle to the electrokinetic response. Finally, one can mention that the asymmetry of the water molecule may induce one on the electrokinetic response. In a silicon nanochannel bearing either a positive or negative charge and containing an $\mathrm{NaF}$ solution, electro-osmotic flows of opposite signs were observed (as expected) but with magnitudes differing by a factor of 3 [92]. This was attributed to the asymmetry of the water molecule, although the asymmetry between ions may also play a role, as discussed below.

\subsection{Hydrodynamics of confined fluid and wettability}

The presence of an interface not only changes the overall dynamics of the solvent, but also its local properties. It is for example well known that the solvent density is not homogeneous and one generally observes the layering of the solvent over a few molecular layers. This change in the structure also has consequences on the electric and hydrodynamic responses, which are generally not accounted for in continuous theories and mesoscopic simulations. In the past few years, significant progress in the understanding of these aspects have been possible thanks to molecular simulations. We discuss first the consequences of confinement on the hydrodynamic behaviour.

When the characteristic length of the confinement (the distance between surfaces in the case of a slit pore) becomes comparable to the molecular size $\sigma$, departure from macroscopic hydrodynamics can be observed. When the channel size exceeds approximately 10 molecular diameters, it is still possible to describe the solvent flow using the Navier-Stokes, provided that the appropriate boundary condition (b.c.) is introduced. Bocquet and Barrat $[40,41,93]$ have shown that the slip b.c.:

$$
\left.\frac{\partial v_{x}}{\partial z}\right|_{z=z_{b}}=\frac{v_{x}\left(z_{b}\right)}{b}
$$

with $x$ and $z$ directions parallel and normal to the wall, respectively, $z_{b}$ the plane where the b.c. applies and $b$ the slip length, generally provides a better description 
and, more importantly, derived Green-Kubo expressions for $z_{b}$ and $b$, thus providing a means to determine the latter from MD simulations. While the convergence of the corresponding integrals is rather slow, the procedure could be validated by comparison with NEMD. A recent study on the slip of water on graphene surfaces highlighted, on the contrary, the limitations of NEMD simulations for large slip [94].

The slip length, which depends on the fluid/wall interaction, is also directly related to the friction at the solid wall. More recently, Huang et al. demonstrated that the slip length is related to the contact angle $\theta$, which is a static quantity, by a simple quasi-universal relationship: $b \propto(1+\cos \theta)^{-2}$ [95]. The slip length is also related to another microscopic quantity, the depletion length, which quantifies the width of the vapour-like region at the solid-liquid interface, as illustrated on Figure 3. Slippage further depends on the roughness of the wall and the possible presence of hydrophilic groups on the surface. The overall hydrophobicity of a surface (as measured by the contact angle) results from the interplay between all these features and it was shown for example that it could be enhanced by coupling the surface polarity and its topography [96]. On hydrophilic surfaces the viscosity of water appears to increase by a factor of 2 to 4 , as a result of intermittent binding of water molecules to binding sites [97].

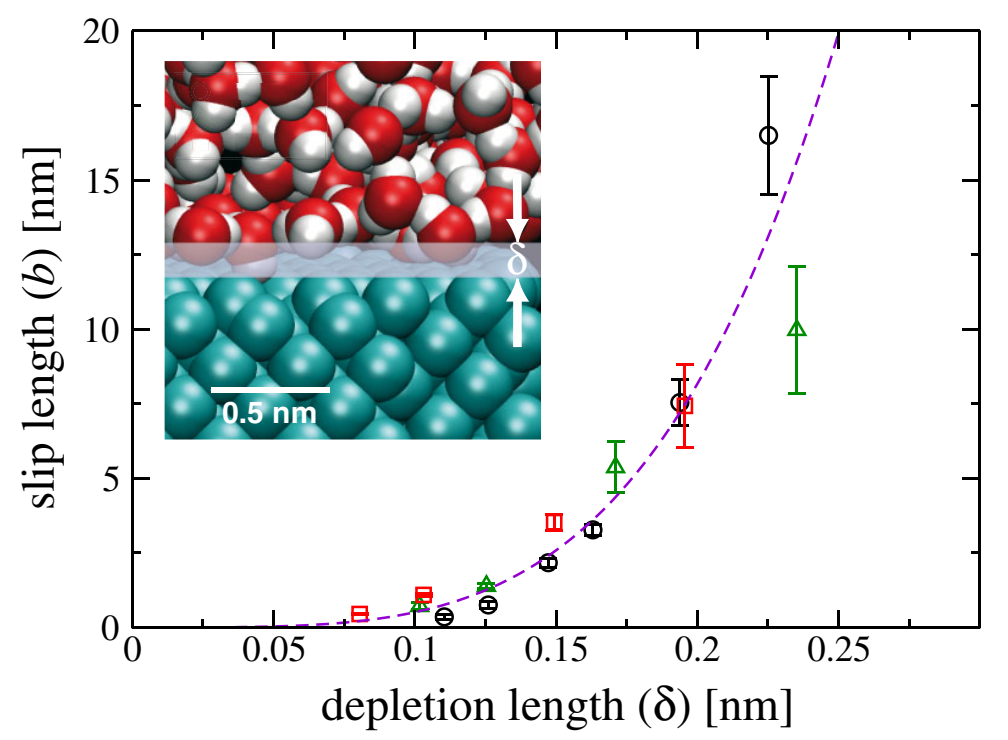

Figure 3. Slip length $b$ as a function of the depletion length $\delta$, which quantifies the width of the vapour-like region at the solid-liquid interface [95]. More hydrophobic surfaces correspond to larger $\delta, b$ and contact angle $\theta$. The dashed line is the scaling prediction $b \propto \delta^{4}$. Reprinted with permission from Huang et al. Phys. Rev. Lett., 101, 226101 (2008). Copyright (2008) by the American Physical Society.

For even stronger confinement (of the order of 10 molecular diameters), one should consider a local viscosity instead of a homogeneous one. However the simple linear relation defining viscosity as the ratio between shear stress and shear rate breaks down [98], as this ratio may even be negative in some regions. In order to overcome these difficulty, one can resort to theories involving a non-local viscosity [99] or determine a local viscosity from the density profiles [100]. To that end, a partitioning between a configurational and translational viscosity is introduced together with a proper weighting scheme [101].

So far, the consequences on electrokinetic effects have been explored only in terms of slip boundary conditions. As an example, Joly et al. determined the slip length in a nano-channel consisting of ions in a Lennard-Jones (LJ) solvent confined between LJ solid walls by appplying an external force and examined the consequence of this 
slip on electro-kinetic flows [47]. On a non-wetting surface (in the studied case, $b \sim 10 \sigma$ ), they found for example that the electro-osmotic velocity (or, equivalently, the $\zeta$-potential) increased by a factor $(1+\kappa b)$, with $\kappa^{-1}$ the Debye length, compared to the wetting case. This prediction has then also been verified experimentally [102]. At a curved surface, this amplication factor was predicted to be $\left(1+\kappa b_{e f f}\right)$, where $b_{\text {eff }}^{-1}=b^{-1}+a^{-1}$ with $a$ the curvature radius. The effect of curvature on the friction has been further clarified by Falk et al., who investigated water flow inside and outside carbon nanotubes [103]. As a result of interactions between mobiles ions and the charged sites on the surface, the slip length decreases with increasing surface charge density $\Sigma$. For a regularly distributed charge, this decrease scales as:

$$
\frac{1}{b(\Sigma)}=\frac{1}{b(\Sigma=0)}+\alpha \frac{a^{2}}{4 \pi \epsilon_{\text {surf }} k_{B} T} \Sigma^{2}
$$

with $\alpha \approx 1$ a numerical prefactor, $a$ a length characterising the surface roughness (typically the atomic diameter) and $\epsilon_{\text {surf }}$ the dielectric constant at the interface, expected to be closer to 1 than in the liquid phase [47]. However, patterning of the surface charges also impacts the overall electrokinetic flow [104].

\subsection{Ionic effects}

Electro-friction When an electric field is applied parallel to the wall, it acts on the dissolved ions which then transfer momentum to the solvent and set the whole fluid in motion. For large surface charge densities, the attraction between dissolved ions and charged surface sites may result in the partial immobilization of a fraction of them. This (electro-)friction and the interactions between ions in the crowded surface layer reduces the electro-osmotic mobility, even if the solvent is not accounted for [105]. Hydrodynamic interactions between ions, even at the continuous solvent (Rotne-Prager) level also contribute to the overall response in the presence of corrugated surfaces [106]. Wu and Aluru further analyzed the same idea using an explicit LJ solvent and determined the effect of ion-wall interactions on Poiseuille and electro-osmotic flows [107]. The intermittent adsorption of ions results in a loss of driving force, which is usually interpreted as an increased fluid solvent viscosity, could contribute significantly to the decrease in the electro-osmotic velocity even though other factors (e.g. hydrogen bonding with the surface) may dominate.

Charge inversion When the surface charge density is large and/or with mutivalent ions, the electro-osmotic flow can be in the direction opposite to the one predicted by the Helmholtz-Smoluchowski theory [108]. While for moderate ion-wall attraction the immobilization of some ions simply results in a loss of driving force, one observe in that case that the charge of condensed counterions overcompensates that of the surface, resulting in an apparent charge inversion. As illustrated in Figure 4, introducing the ion density profiles obtained by MD simulations to predict the forcing term in the Navier-Stokes equation allows to account for this charge reversal, but not to quantitatively reproduce the EOF profile [66]. The charge inversion disappears in the presence of a large concentration of monovalent ions $[109,110]$, as observed experimentally.

Ion-specificity On a hydrophobic surface, the electrokinetic response is very sensitive to the nature of the ions present in the fluid: isovalent ions with different ionic sizes will give rise, for example, to different electro-osmotic flows. Huang et $a l$. have shown that this behaviour can be explained by the different affinity of ions for the "vapour-liquid-like" interface at such walls, as a function of their ionic size 

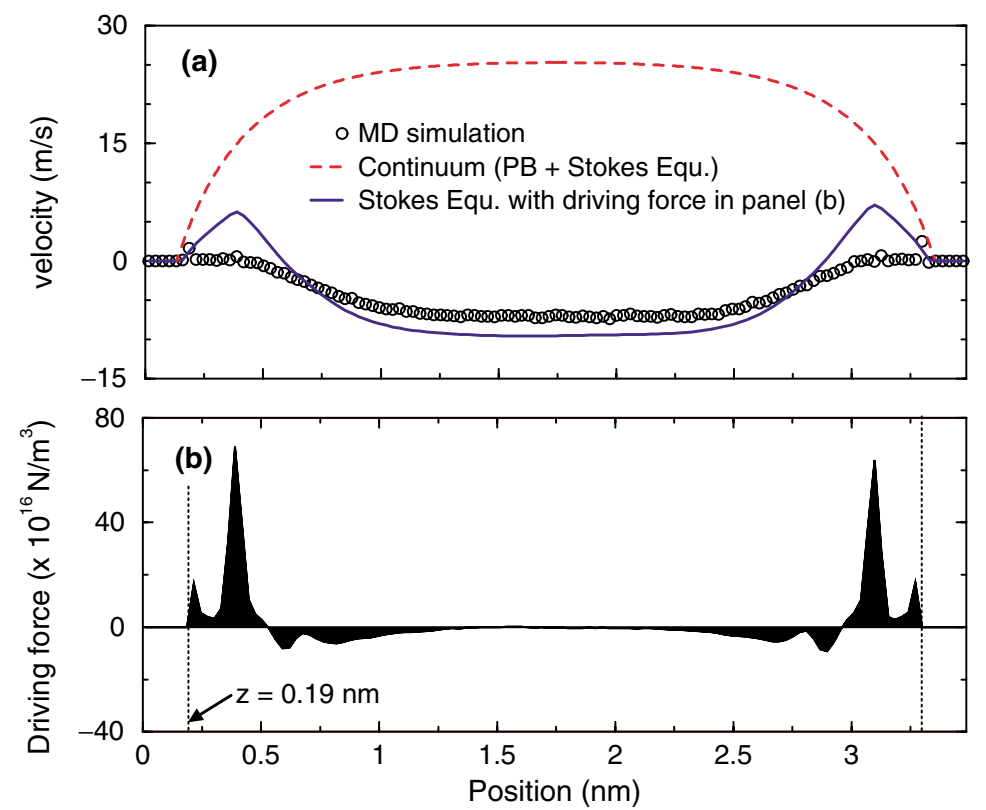

Figure 4. (a) Electro-osmotic velocity profile in a highly charged slit pore containing an aqueous solution, from molecular simulations and from continuous theories. The solid line is the solution of the Stokes equation using the local force from molecular simulation, which is indicated in (b). The electro-osmotic flow is in the direction opposite to the one expected from the sign of the surface charge, because of the condensation of counterions results in a charge inversion [66]. Reprinted with permission from Qiao and Aluru, Phys. Rev. Lett., 92, 198301 (2004). Copyright (2004) by the American Physical Society.

(increasing affinity with ionic size). This preferential location of ions near the surface also explains the possibility of non-zero $\zeta$-potentials (or equivalently non-zero EOF) at an uncharged hydrophobic wall [111]. Huang et al. further proposed to introduce this ion-specificity in a modified Poisson-Boltzmann description, based on the partial desolvation of the interfacial ion which then experiences a dielectric discontinuity (hence forces from image charges) and the interaction with the wall [111]. This simple model proved sufficient to capture most of the molecular simulation results [112]. A similar model was shown by Schwierz et al. to also account for the interplay between surface charge and polarity on the adsorption of anions, for which reversed Hofmeister series have been reported on hydrophilic negative surfaces and hydrophobic positive ones [113]. These findings also have implications for the electrokinetic behaviour of these surfaces. Finally, in surfaces with more complex chemical moities, such as the silica surface, the molecular nature and its hydrogen bonding to the surface may also play a role in ion-specific effects, beyond the mere dielectric discontinuity [114].

\section{Focus on recent applications}

\subsection{Colloids and polyelectrolytes}

The electrokinetic behaviour of suspensions of colloids or polyelectrolytes is sensitive to the hydrodynamics of the solvent and its coupling to the dissolved ions. Since the size of the colloids and polymers, ranging from nano- to micrometers, is large compared with the solvent and typical ion molecular sizes, the standard theory, restricted to the linear regime, neglects ion correlations and treats them in terms of continuous density distributions. If the double-layers are much narrower than the macromolecular size, it is even reasonable to neglect the distortion 
of the charge distribution by the flow (induced by an applied electric field or a gravitational acceleration). Then, analytic expressions for the electrophoretic or sedimentation velocities of charged colloids are known. Beyond this regime, the standard theory describing the electrophoresis of charged macro ions was developed by O'Brien and White [115]. LB has been used to analyze colloidal electrophoresis outside this linear regime and analyze the impact of strong electrostatic forces on the electrophoretic mobility, as displayed in Figure 5. The possibility to tune ion diffusivity has also allowed to address the relevance of the Péclet number in the effective mobility [116] and shown that the decrease in diffusivity affects both the mean electrophoretic velocity and its dispersion. A systematic study in the nonlinear regime requires a proper identification of the relationship between the colloidal charge, its effective charge outside equilibrium and its relation with the effective $\zeta$-potential; numerical simulations provide a systematic means to quantify the relation between these various quantities [117].

Studies with resolved ions in LB have also been used to study the electrophoresis of suspensions in the linear regime. Making use of the Green-Kubo relation [118], it has been possible to derive the electrophoretic mobility in the linear regime as a function of the salt concentration and charge magnitude, moving also beyond the standard regime. By analyzing the limit in which there is no salt (only counterions), Lobaskin et. al. were also able to address the role of screening in the electrophoretic mobility of colloidal suspensions [119]. The limit of the electrophoretic mobility in dense suspensions has also been addressed using the Smooth Profile method (SP), which combines a Navier-Stokes solver in which colloids are treated through a field that localizes the colloids' position [120]. This study demonstrated that with strong fields or wide double-layers the interaction between colloids through the distorted double-layers leads to pattern formations and favours the alignment of moving colloids. The analysis of the sedimentation velocity of colloids has helped to show the relevance of the colloidal shape in the electrokinetic response [31].

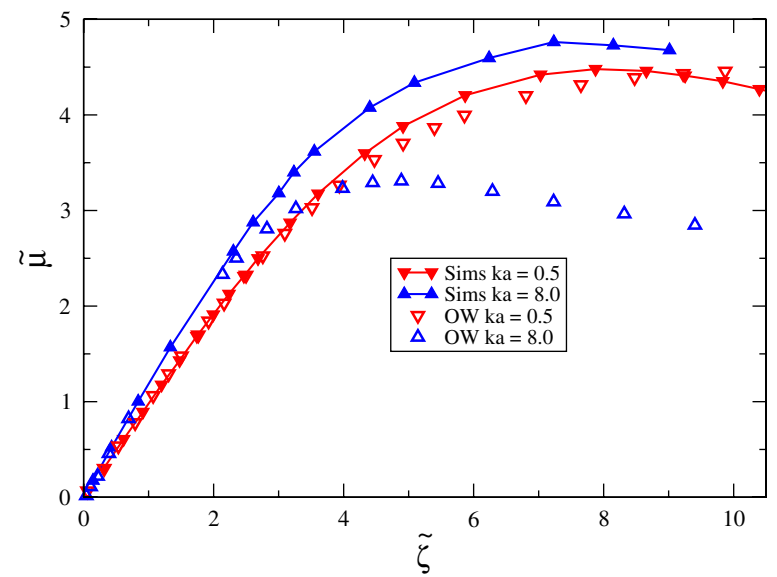

Figure 5. Electrophoretic mobility of a charged colloid subject to a uniform applied electric field as a function of the colloid charge, expressed in terms of the dimensional surface potential $\tilde{\zeta}$, for different values of the ratio between the colloid radius and the Debye length, $\kappa a$ [116]. Symbols correspond to Lattice-Boltzmann Electrokinetics simulations, while solid lines correspond to the theoretical prediction of O'Brien and White [115]. Reprinted with permission from Giupponi and Pagonabarraga, Phys. Rev. Lett., 106, 248304 (2011). Copyright (2011) by the American Physical Society.

Within the linearized, Debye-Hückel regime, there have also been efficient proposals to introduce the coupling between a charged particle and the electrolyte in which it is embedded, in order to reach larger systems and longer time scales [121]. This approximate approach can be competitive for the study of the electrokinetics 
of polyelectrolytes [121]. Such a strategy is complementary to more detailed simulations carried out on single polyelectrolyte chains to understand the different regimes that characterize their electrophoresis, at low salt concentrations, which distinguish polyelectrolyte electrokinetic behaviour from the ones known for charged colloids. Polyelectrolyte electrophoretic mobilities have also been analyzed using resolved ions in a continuous solvent, using a variant of LB. The study demonstrated the relevance of the polymer size for the chain mobility [122]. In the presence of multivalent ions, the structure of the dissolved electrolyte around the chain is more complex and it affects the response of the system to imposed fields. In this case, MD simulations have been performed in order to address the relevance of ion correlations, their impact on the accumulation of ions around the chain and on the measured effective mobility [123].

\subsection{Electrokinetics at clay surfaces}

Clay minerals are layered aluminosilicates present in large quantities in soils and in the underground. They play an important role in many environmental and industrial processes and have therefore attracted a lot of attention from the molecular simulation community since two decades. Most of these minerals bear a negative charge due to substitutions in the mineral framework (e.g. $\mathrm{Mg}^{2+}$ for $\mathrm{Al}^{3+}$ or $\mathrm{Al}^{3+}$ for $\mathrm{Si}^{4+}$ ) which is compensated by cations at their surface. Electrophoretic mobility measurements are often used to estimate their surface charge density. However the connection between these two quantity is not straightforward since it relies on a number of debatable assumptions on the shape of the particles and the hydrodynamic boundary conditions to consider.

Marry et al. introduced a Green-Kubo formula for the electro-osmotic response and used equilibrium molecular simulations to determine the electro-osmotic velocity profile between montmorillonite surfaces separated by thin water films (from 2 to $4 \mathrm{~nm}$ ) [42, 43, 124]. Although the convergence of the Green-Kubo integrals was rather slow, they found that these could only be explained if slip boundary conditions were introduced, even though with a small slip length, as illustrated in Figure 6. They also discussed the consequences of this slip on the EOF within the framework of Poisson-Boltzmann theory and showed that the latter was not sufficiently accurate to reproduce the ionic profiles, hence the EOF. More recently, NEMD simulations of Poiseuille flows in the same system allowed to address the validity of continuous hydrodynamic description as a function of the distance between surfaces [49]. The velocity profile in the first water layers could not be reproduced by introducing a local viscosity from the ratio between shear stress and shear rate, as this resulted in negative values. It was proposed instead to use a simplified picture of a homogeneous fluid with the bulk density and viscosity and to apply a slip boundary condition at the Gibbs Dividing Surface, with a slip length determined from molecular simulation $(\approx 2 \AA)$. Such a description was shown to be reasonably accurate for pore sizes larger than $4.5 \mathrm{~nm}$, but not applicable for smaller pores in which the density is inhomogeneous throughout the fluid.

The same group also reported a detailed study of water on montmorillonite and analyzed the structure and dynamics of the hydrogen bond network on that surface $[125,126]$. It was found that the formation and breaking of hydrogen bonds between molecules in the first liquid layer and the surface, as well as the departure of molecules from that layer, are reasonably well described by first order kinetics. Moreover, diffusion in the direction parallel to the surface is slowed down compared to that in the bulk. Comparing $\mathrm{Cs}^{+}$and $\mathrm{Na}^{+}$counterions, it was observed that the nature of the cation had a limited influence on the diffusion coefficient of surface 


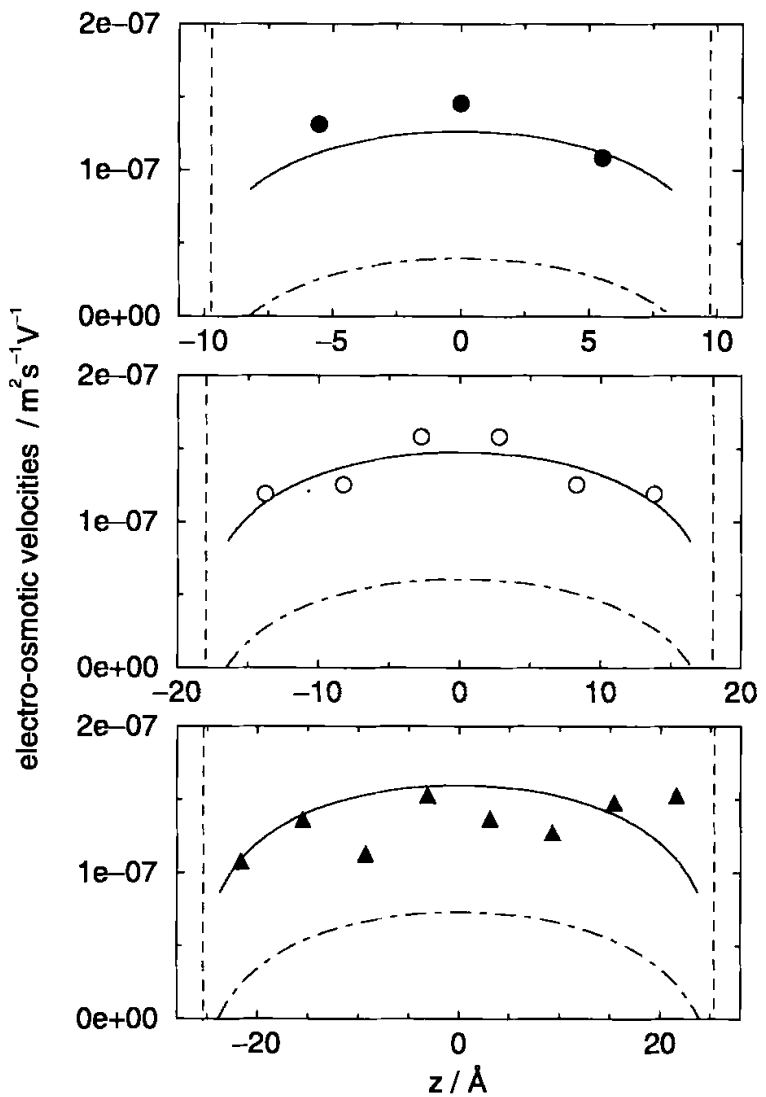

Figure 6. Electro-osmotic velocity profiles in a montmorillonite clay slit pore (for 2,3 and $4 \mathrm{~nm}$ widths, from top to bottom). The symbols are molecular dynamics results from equilibrium simulations, using a Green-Kubo expression. The solution from the Poisson-Boltzmann and Stokes equations can reproduce the MD results only if a slip boundary condition (solid lines) is introduced [42]. Reprinted with permission from Marry et al., Mol. Phys., 101, 3111 (2003). Copyright (2003) by Taylor and Francis.

water molecules and on the kinetic rates for the formation and breaking of hydrogen bonds with the surface.

Several studies have since then improved our understanding of the behaviour of water on such surfaces. In particular, it was possible to disentangle the role of ions from that of the water-surface interactions. To that end, the study of the electrically neutral clay analogue of montmorillonite, namely pyrophyllite, and similar clay minerals (talc, in which a surface hydroxyl group which is parallel to the surface in pyrophyllite is perpendicular to the surface, and fluorotalc in which this group is replaced by a fluorine atom) were investigated both in the low relative humidity and high relative humidity regimes [127]. It was then found that in the absence of ions, all three surfaces display an adsorption site for water molecules, which is very weak for talk and pyrophyllite and strong for talc owing to the formation of a hydrogen bond. In all cases, however, binding to the surface is weaker than the cohesion between water molecules so that at saturation the binding sites are empty and the surface behaves as hydrophobic.

Finally, Botan et al. simulated Poiseuille and electro-osmotic flows in clay nanopores using NEMD [48]. They showed that, using the slip or stagnation length (depending on the clay type and force field used) determined from Poiseuille flows and the ionic density profiles from MD simulations, the Navier-Stokes equation is able to reproduce the simulated EOF. As previously shown, the Poisson-Boltzmann theory is not sufficient to predict the ionic distribution, hence the velocity profile. The behaviour predicted by the most reliable force field (clayFF) is a hydrophobic 
(slip) pyrophyllite and a hydrophilic (stick) montmorillonite. The role of the clay charge and of cations on surface water molecules was investigated by comparing Poiseuille flows on montmorillonite (charged with counterions) on the one hand and pyrophyllite (same structure but uncharged) in the presence on an applied electric field perpendicular to the surface, with a magnitude corresponding to the surface charge density of pyrophyllite, on the other hand. The average electric field created by the charged surface explains most of the effect on surface water and the presence of cations at the surface only plays a secondary role. Finally, by comparing different force fields, the effect of the polarity of the surface on hydrogen bond strength could be analyzed.

\subsection{Electrokinetics with ionic liquids}

Room-temperature ionic liquids (RTIL) are liquids consisting solely of ions, often organic ones with a low melting temperature (compared to usual molten salts). The structure of the liquid at a surface is difficult to probe experimentally, although recent surface-sensitive spectroscopic techniques such as SFG have recently allowed to observed interesting features such as a charge inversion for a RTIL at different salt surfaces [128]. Surface Force Balance also have brought insights into the layered structure and the friction of ionic liquids confined between charged surfaces $[129,130]$. Most of the understanding of the microscopic structures comes from theoretical predictions and molecular simulations. In particular, electrostatic and steric correlations between ions result in a very structured interface where the density of both cations and anions display oscillations. Although molecular simulations are necessary to make quantitative predictions, given the complexity of interactions between ions, Kornyshev introduced a relatively simple model capturing how ion correlations and molecular crowding produce an interface which radically departs form the double-layer picture appropriate in the presence of a solvent [131]. One might thus expect dramatic consequences for the dynamic response of such fluids under the effect of an applied electric field.

Jiang and Qiao recently reported a molecular dynamics study of electrokinetic flow in a RTIL confined between charged walls [132]. The layered structure of the fluid, which displays oscillations in the electric charge as a function of the distance to the wall, induces body forces varying over molecular distances inside the fluid, which eventually result in an oscillatory EOF, as illustrated in Figure 7. The direction of the EOF in the center of the pore is opposite to the one expected from the sign of the surface charge, owing to the charge inversion. The latter is often referred to as overscreening in the context of ions at charged surfaces and results from the larger charge within the first fluid layer than required to compensate that of the surface. This flow reversal can be explained by the strong interactions between ions in that first layer with the wall, with a significant electro-friction and a loss of driving of the fluid in that direction. The next layer of ions is more mobile and can drive the fluid more efficiently.

These authors then proposed a generalized hydrodynamics framework to describe these effects in a continuous approach, calibrated on molecular simulations. In order to capture the effect of ion-ion correlations on short length scales, they determined the generalized viscosity $\eta(k)$, which is the Fourier transform of the viscosity kernel $\eta\left(z-z^{\prime}\right)$ linking the stress tensor $\tau_{z x}$ at a given position to the shear stress $\dot{\gamma}$ at all other positions:

$$
\tau_{z x}(z)=\int_{-\infty}^{\infty} \eta\left(z-z^{\prime}\right) \dot{\gamma}\left(z^{\prime}\right) \mathrm{d} z^{\prime}
$$




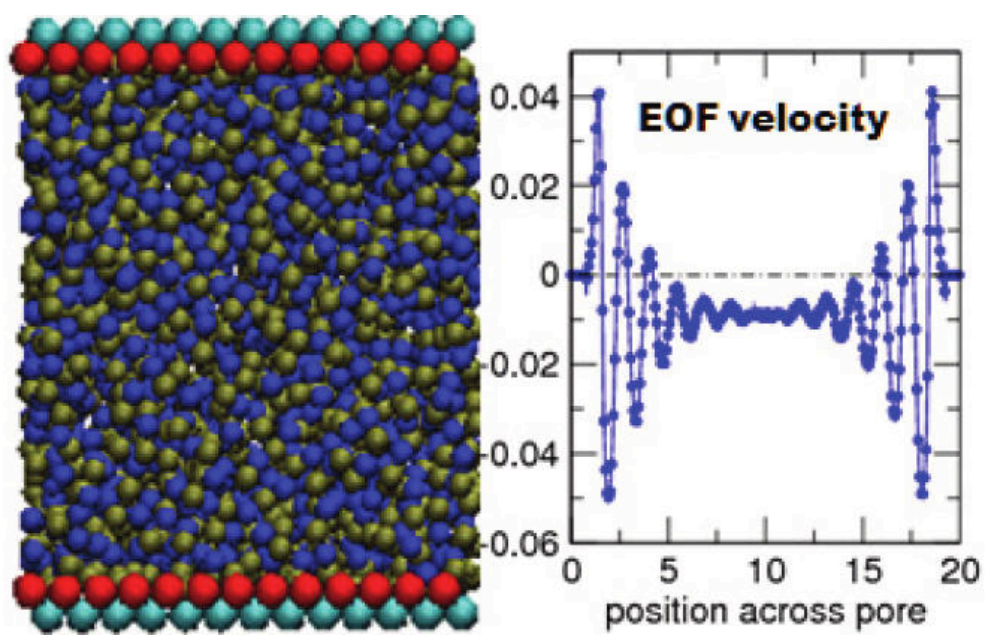

Figure 7. The electro-osmotic flow in a room temperature ionic liquid (RTIL) between charged surfaces displays oscillations, as a result of strong ionic correlations [132]. In such liquids, short-wavelength hydrodynamics profoundly affects the macroscopic electro-osmotic flow. Reprinted with permission from Jiang and Qiao, J. Phys. Chem. C, 116, 1133 (2012). Copyright (2012) American Chemical Society.

The generalized viscosity $\eta(k)$ could be fitted to a Gaussian and it was then possible to reproduce the simulation data for the shear stress and the EOF. These results demonstrate the importance of accounting for short-wavelength hydrodynamics for the description of EOF in RTILs, as the classical hydrodynamic theories (with a local viscosity) cannot capture the effect of short-range ionic correlations.

\subsection{Electrokinetics without mobile charges}

Let us conclude this section by evoking the interesting issue of electrokinetics without mobile charges, which was debated in the last few years. This question was raised in the context of understanding the electrophoretic mobility of air bubbles and oil droplets. The fact that such neutral systems display such a mobility may seem surprising. Several explanations have been proposed, including the generally accepted idea of ion adsorption (including possibly hydroxyl $\mathrm{OH}^{-}$and hydronium $\mathrm{H}_{3} \mathrm{O}^{+}$ions) or the asymmetric structure of the water-air interface. An alternative mechanism has been proposed by Knecht et al. who found, using NEMD simulations a finite mobility for a heptane droplet in water [133]. Similarly, Joseph and Aluru reported the possibility of pumping water molecules through carbon nanotubes with a static electric field and proposed a mechanism based a translationalrotation coupling for interfacial water molecules [134].

These results contradict Onsager's reciprocity theorem which implies that free charge must be present to generate an electric current from a pressure gradient. It was then showed that these simulation results were an artifact of the implementation of the simple truncation of short range interactions in the GROMACS software [135]. Moreover, Bonthuis et al. demonstrated in a generalized NavierStokes approach that such a coupling cannot be exploited with a static field, but that pumping was possible using a rotating field in an asymmetric channel [136]. Finally, Rinne et al. showed that it was possible to pump water through carbon nanotubes using electrodes with charges oscillating in the $\mathrm{GHz}$ range, by introducing a phase shift between the electrodes to break the spatio-temporal symmetry [137]. While in practice virtually all current applications of electrokinetics involve mobile charges, these studies bring fundamental insights into the microscopic coupling between charge and mass flows. 


\section{Conclusion and perspectives}

Electrokinetic phenomena involve a range of length and time scales, from the molecular to the macroscopic ones, and their description requires a multiscale strategy combining different simulation techniques. In the present review, we have first addressed the various mesoscopic approaches proposed recently, which vary in their explicit/implicit treatment of the solvent and microions. In particular, we have emphasized the recent progress achieved with the lattice methods based on kinetic theory, which offer a range of convenient simulation tools for the simulation of complex charged fluids in arbitrary geometries, including under extreme confinement. We have then focussed our discussion on the insights on electrokinetic effects gained from molecular simulations, which have been extremely useful in this field over the past decade, with a very active community in the last few years. We finally illustrated recent applications of these methods.

We anticipate that both mesoscale and molecular simulations will continue to develop in the future, as they address complementary issues in a context which requires a description of both short and long-ranged phenomena. For example, one aspect on the molecular scale which is known to play an important role on the structure of the interface but whose effect on electrokinetic properties has not been investigated is that of polarizability, in particular the electric response of water molecules to the anisotropic environment they experience at an interface. Ab-initio simulations will be useful to determine the origin of the surface charge in new materials which exploit electrokinetic effects, such as in the recent experimental and theoretical study of a single transmembrane boron nitride nanotube by Siria et al. [138]. Another challenge for the modelling of electrokinetics is the description of proton transport near interfaces, e.g. in membranes [139-141], at the surface of metal electrodes [142, 143], of minerals [144] or of biological molecules [145], and for which quantum effects must be accounted for.

On the mesoscopic scale, efforts should be devoted to the improvement of free energy based descriptions to go beyond mean-field descriptions and capture e.g. the effect of ionic correlations on both the thermodynamical and dynamical properties. The insight gained by these new techniques, together with the possibility to address in detail the interplay between ion specificity and the collective response of charged fluids, opens new venues to contrast with recent theoretical developments since simulations can be more easily used to test the theoretical approximations and falsify their predictions. From the computational view, the community should greatly benefit from the development of standard open-source codes for mesoscale simulations, such as the ones that have emerged in the ab-initio (e.g. $[146,147])$ or classical MD (e.g. $[148,149])$ communities. Examples of such software include the ESPResSo [150] or the DL_MESO [151] simulation packages.

In the future, we believe that the multiscale strategy will not be limited to the simulation on different scales. In fact, one already observes in many contexts the generalization of bottom-up strategies whereby each level of description is calibrated on a more fundamental one. The challenge is then to keep track of the relevant degrees of freedom while averaging them out. An example of this strategy for electrokinetics can be found in Ref. [152], but other works discussed here, where continuous models are calibrated on molecular simulations, also fall in this category. Another approach to bridge scales is that of hybrid simulations in which a molecular simulation is explicitly coupled to a coarser level of description such as continuum hydrodynamics. This allows to correctly capture the complex momentum and energy transfer occurring at a boundary while keeping a simple description further away from the interface [153]. Such hybrid approaches may also 
be well suited to capture the effect of thermal fluctuations, which are known to play an important role in nanofluidic transport [154]. In that respect, fluctuating hydrodynamics appears as a promising method to couple a molecular description with a mesoscopic one while explicitly accounting for thermal flucuations [155157], although the introduction of ionic species and the corresponding long-range electrostatic interactions remains necessary for the description of electrokinetic phenomena. Similarly, the recent progress in the understanding of the fluctuating Lattice-Boltzmann method [158] suggests that it can also be adapted to capture thermal fluctuations at this level, including for complex fluids $[159,160]$, even though it has not yet been applied to the case of charged fluids.

\section{Acknowledgements}

B.R. acknowledges financial support from the Agence Nationale de la Recherche under grant ANR-09-SYSC-012. I.P. acknowledges Direccion General de Investigacion (Spain) and DURSI for financial support under Projects No. FIS 2008-04386 and No. 2009SGR-634, respectively.

\section{References}

[1] L. Bocquet and E. Charlaix, Chemical Society Reviews 39 (3), 1073 (2010).

[2] W. Sparreboom, A. Van Den Berg and J.C.T. Eijkel, New Journal of Physics 12 (1), 015004 (2010).

[3] K. Kang and J.K.G. Dhont, Soft Matter 6 (2), 273 (2010).

[4] J.K.G. Dhont and K. Kang, The European Physical Journal E 34 (4), 1 (2011).

[5] P. Papadopoulos, X. Deng, D. Vollmer and H.J. Butt, Journal of Physics: Condensed Matter 24 (46), 464110 (2012).

[6] L.C. Chen, C.C. Wu, R.G. Wu and H.C. Chang, Langmuir 28 (31), 11281 (2012).

[7] B.D. Storey and M.Z. Bazant, Physical Review E 86 (5), 056303 (2012).

[8] T.L. Horng, T.C. Lin, C. Liu and B. Eisenberg, The Journal of Physical Chemistry B (2012).

[9] M.Z. Bazant, M.S. Kilic, B.D. Storey and A. Ajdari, Advances in Colloid and Interface Science 152 (1-2), 48 (2009).

[10] L. Samaj and E. Trizac, Physical Review Letters 106 (7), 078301 (2011).

[11] I. Pagonabarraga, B. Rotenberg and D. Frenkel, Phys. Chem. Chem. Phys. 12 (33), 9566 (2010).

[12] J. Rotne and S. Prager, J. Chem. Phys. 50, 4831 (1969).

[13] J.P. Hernandez-Ortiz, J.J. de Pablo and M.D. Graham, Phys. Rev. Lett. 98, 140602 (2007).

[14] P.J. Hoogerbrugge and J.M.V.A. Koelman, Europhys. Lett. 19, 155 (1992).

[15] P. Español and P.B. Warren, Europhys. Lett. 30, 191 (1995).

[16] C. Lowe, Europhys. J. 47, 145 (1999).

[17] P. Español and M. Revenga, Phys. Rev. E 67, 026705 (2003).

[18] R.D. Groot, J. Chem. Phys. 118, 11265 (2003).

[19] J. Smiatek and F. Schmid, Comp. Phys. Comm. 182, 1941 (2011).

[20] W.B. Russel, D. Saville and W.R. Schowalter, Colloidal Dispersions (, , 1989).

[21] V. Dahirel and M. Jardat, Current Opinion in Colloid \& Interface Science 15 (1-2), 2 (2010).

[22] A. Ajdari and D. Long, Europ. J. Phys. E 4, 29 (2001).

[23] M. Rex and H. Löwen, Europ. J. Phys. E 26, 143 (2008).

[24] S. Succi, The Lattice Boltzmann Equation for Fluid Dynamics and Beyond (, , 2001).

[25] B. Dünweg and A. Ladd, Adv. Pol. Sci. 221, 89 (2009).

[26] M. Cates, K. Stratford, R. Adhikari, P. Stansell, J.C. Desplat, I. Pagonabarraga and A. Wagner, J. Phys.: Cond. Mat. 16, S3903 (2004).

[27] K. Stratford and I. Pagonabarraga, Comp. and Math. with Appl. 55, 1585 (2008).

[28] U. Marini Bettolo Marconi and S. Melchionna, The Journal of Chemical Physics 131 (1), 014105 (2009).

[29] F. Capuani, I. Pagonabarraga and D. Frenkel, The Journal of Chemical Physics 121 (2), 973 (2004). 
[30] I. Pagonabarraga, F. Capuani and D. Frenkel, Computer physics communications 169 (1), $192(2005)$.

[31] F. Capuani, I. Pagonabarraga and D. Frenkel, The Journal of Chemical Physics 124 (12), 124903 (2006).

[32] J. Smiatek, M. Sega, C. Holm, U. Schiller and F. Schmid, J. Chem. Phys. 130, 244702 (2009).

[33] S. Melchionna and U.M.B. Marconi, EPL (Europhysics Letters) 95 (4), 44002 (2011).

[34] U. Marini Bettolo Marconi and S. Melchionna, Langmuir 28 (38), 13727 (2012).

[35] R. Schmitz and B. Dünweg, Journal of Physics: Condensed Matter 24 (46), 464111 (2012).

[36] C. Lowe, D. Frenkel and M. Van der Hoef, J. Stat. Phys. 87, 1129 (1997).

[37] B. Rotenberg, I. Pagonabarraga and D. Frenkel, EPL (Europhysics Letters) 83 (3), 34004 (2008).

[38] D. Moroni, B. Rotenberg, J.P. Hansen, S. Succi and S. Melchionna, Phys. Rev. E 73, 066707 (2006).

[39] B. Rotenberg and D. Moroni, Phys. Rev. E 74, 037701 (2006).

[40] L. Bocquet and J.L. Barrat, Physical Review E 49 (4), 3079 (1994).

[41] L. Bocquet and J.L. Barrat, Journal of Physics: Condensed Matter 8 (47), 9297 (1996).

[42] V. Marry, J.F. Dufrêche, M. Jardat and P. Turq, Molecular Physics 101 (20), 3111 (2003).

[43] J.F. Dufrêche, V. Marry, N. Malikova and P. Turq, Journal of molecular liquids 118 (1), $145(2005)$

[44] J.B. Freund, The Journal of Chemical Physics 116 (5), 2194 (2002).

[45] R. Qiao and N.R. Aluru, The Journal of Chemical Physics 118 (10), 4692 (2003).

[46] Y. Wang, Y. Wang, K. Chen and B. Li, Chemical Engineering Science 66 (12), 2807 (2011).

[47] L. Joly, C. Ybert, E. Trizac and L. Bocquet, The Journal of Chemical Physics 125 (20), 204716 (2006).

[48] A. Botan, V. Marry, B. Rotenberg, P. Turq and B. Noetinger, The Journal of Physical Chemistry C 117 (2), 978 (2013).

[49] A. Botan, B. Rotenberg, V. Marry, P. Turq and B. Noetinger, The Journal of Physical Chemistry C 115 (32), 16109 (2011).

[50] D. Frenkel and B. Smit, Understanding Molecular Simulations, From Algorithms to Applications (, , 2002).

[51] R. Hockney and J. Eastwood, Computer Simulation Using Particles (, , 1981).

[52] B. Dünweg and K. Kremer, J. Chem. Phys. 99, 6983 (2004).

[53] I.C. Yeh and G. Hummer, J. Phys. Chem. B 108 (40), 15873 (2004).

[54] S. Tazi, A. Botan, M. Salanne, V. Marry, P. Turq and B. Rotenberg, Journal of Physics: Condensed Matter 24 (28), 284117 (2012).

[55] D.J. Bonthuis, K.F. Rinne, K. Falk, C.N. Kaplan, D. Horinek, A.N. Berker, L. Bocquet and R.R. Netz, Journal of Physics: Condensed Matter 23 (18), 184110 (2011).

[56] Y. Li, J. Xu and D. Li, Microfluidics and nanofluidics 9 (6), 1011 (2010).

[57] K. Leung, I.M.B. Nielsen and L.J. Criscenti, Journal of the American Chemical Society 131 (51), 18358 (2009).

[58] M. Sulpizi and M. Sprik, Journal of Physics: Condensed Matter 22 (28), 284116 (2010).

[59] M.P. Gaigeot, M. Sprik and M. Sulpizi, Journal of Physics: Condensed Matter 24 (12), 124106 (2012).

[60] M. Sulpizi, M.P. Gaigeot and M. Sprik, Journal of Chemical Theory and Computation 8 (3), 1037 (2012).

[61] S. Tazi, B. Rotenberg, M. Salanne, M. Sprik and M. Sulpizi, Geochimica et Cosmochimica Acta 94 (0), 1 (2012).

[62] R.R. Netz, Current Opinion in Colloid and Interface Science 9 (1), 192 (2004).

[63] P. Jungwirth and D.J. Tobias, Chemical Reviews 106 (4), 1259 (2006).

[64] P. Jungwirth and B. Winter, Annu. Rev. Phys. Chem. 59, 343 (2008).

[65] D. Horinek and R.R. Netz, Physical review letters 99 (22), 226104 (2007).

[66] R. Qiao and N.R. Aluru, Physical Review Letters 92 (19), 198301 (2004).

[67] J. Noah-Vanhoucke and P.L. Geissler, Proceedings of the National Academy of Sciences 106 (36), 15125 (2009).

[68] D.E. Otten, P.R. Shaffer, P.L. Geissler and R.J. Saykally, Proceedings of the National Academy of Sciences 109 (3), 701 (2012).

[69] M. Takahashi, The Journal of Physical Chemistry B 109 (46), 21858 (2005).

[70] R. Vacha, D. Horinek, M.L. Berkowitz and P. Jungwirth, Phys. Chem. Chem. Phys. 10 (32), 4975 (2008).

[71] B. Winter, M. Faubel, R. Vacha and P. Jungwirth, Chemical Physics Letters 474 (4), 241 (2009). 
[72] T.D. Kühne, T.A. Pascal, E. Kaxiras and Y. Jung, J. Phys. Chem. Lett 2, 105 (2011).

[73] R. Vacha, O. Marsalek, A.P. Willard, D.J. Bonthuis, R.R. Netz and P. Jungwirth, The Journal of Physical Chemistry Letters 3 (1), 107 (2011).

[74] S.M. Kathmann, I.F.W. Kuo, C.J. Mundy and G.K. Schenter, The Journal of Physical Chemistry B 115 (15), 4369 (2011).

[75] D.J. Bonthuis, S. Gekle and R.R. Netz, Physical Review Letters 107 (16), 166102 (2011).

[76] D.J. Bonthuis, S. Gekle and R.R. Netz, Langmuir 28 (20), 7679 (2012).

[77] S. Gekle and R.R. Netz, The Journal of Chemical Physics 137 (10), 104704 (2012).

[78] D.J. Bonthuis and R.R. Netz, Langmuir 28 (46), 16049 (2012).

[79] D. Chandler, Physical Review E 48 (4), 2898 (1993).

[80] A.J. Patel, P. Varilly and D. Chandler, The Journal of Physical Chemistry B 114 (4), 1632 (2010).

[81] R. Godawat, S.N. Jamadagni and S. Garde, Proceedings of the National Academy of Sciences 106 (36), 15119 (2009).

[82] A.P. Willard and D. Chandler, The Journal of Physical Chemistry B 114 (5), 1954 (2010).

[83] D. Laage and J.T. Hynes, Science 311 (5762), 832 (2006).

[84] G. Stirnemann and D. Laage, The Journal of Physical Chemistry Letters 1 (10), 1511 (2010).

[85] D. Laage, G. Stirnemann, F. Sterpone and J.T. Hynes, Accounts of Chemical Research 45 (1), 53 (2012).

[86] D. Laage, G. Stirnemann and J.T. Hynes, The Journal of Physical Chemistry B 113 (8), 2428 (2009).

[87] G. Stirnemann, P.J. Rossky, J.T. Hynes and D. Laage, Faraday Discussions 146 (0), 263 (2010).

[88] G. Stirnemann, S.R.V. Castrillon, J.T. Hynes, P.J. Rossky, P.G. Debenedetti and D. Laage, Physical Chemistry Chemical Physics 13 (44), 19911 (2011).

[89] F. Sedlmeier, Y. von Hansen, L. Mengyu, D. Horinek and R.R. Netz, Journal of Statistical Physics 145 (2), 240 (2011).

[90] D. Laage and J.T. Hynes, Proceedings of the National Academy of Sciences 104 (27), 11167 (2007).

[91] K.J. Tielrooij, S.T. Van Der Post, J. Hunger, M. Bonn and H.J. Bakker, The Journal of Physical Chemistry B 115 (43), 1263812647 (2011).

[92] R. Qiao and N.R. Aluru, Applied Physics Letters 86 (14), 143105 (2005).

[93] L. Bocquet and J.L. Barrat, Soft Matter 3 (6), 685693 (2007).

[94] S.K. Kannam, B.D. Todd, J.S. Hansen and P.J. Daivis, The journal of chemical physics 136, 024705 (2012).

[95] D.M. Huang, C. Sendner, D. Horinek, R.R. Netz and L. Bocquet, Physical review letters 101 (22), 226101 (2008).

[96] N. Giovambattista, P.G. Debenedetti and P.J. Rossky, Proceedings of the National Academy of Sciences 106 (36), 15181 (2009).

[97] C. Sendner, D. Horinek, L. Bocquet and R.R. Netz, Langmuir 25 (18), 10768 (2009).

[98] K.P. Travis, B.D. Todd and D.J. Evans, Physical Review E 55 (4), 4288 (1997).

[99] J. Zhang, B.D. Todd and K.P. Travis, The Journal of Chemical Physics 121 (21), 10778 (2004).

[100] H. Hoang and G. Galliero, The Journal of Chemical Physics 136 (12), 124902 (2012).

[101] H. Hoang and G. Galliero, Physical Review E 86 (2), 021202 (2012).

[102] C.I. Bouzigues, P. Tabeling and L. Bocquet, Physical review letters 101 (11), 114503 (2008).

[103] K. Falk, F. Sedlmeier, L. Joly, R.R. Netz and L. Bocquet, Nano Letters 10 (10), 4067 (2010).

[104] H.A. Zambrano, M. Pinti, A.T. Conlisk and S. Prakash, Microfluidics and Nanofluidics pp. 1-13 (2012).

[105] R.R. Netz, Physical Review Letters 91 (13), 138101 (2003).

[106] Y.W. Kim and R.R. Netz, The Journal of Chemical Physics 124 (11), 114709 (2006).

[107] P. Wu and R. Qiao, Physics of Fluids 23, 072005 (2011).

[108] F.H.J. van der Heyden, D. Stein, K. Besteman, S.G. Lemay and C. Dekker, Physical Review Letters 96 (22), 224502 (2006).

[109] C.D. Lorenz and A. Travesset, Physical Review E 75 (6), 061202 (2007).

[110] Y. Chen, Z. Ni, G. Wang, D. Xu and Li, Nano Letters 8 (1), 42 (2008).

[111] D.M. Huang, C. Cottin-Bizonne, C. Ybert and L. Bocquet, Physical review letters 98 (17), 177801 (2007).

[112] D.M. Huang, C. Cottin-Bizonne, C. Ybert and L. Bocquet, Langmuir 24 (4), 1442 (2008).

[113] N. Schwierz, D. Horinek and R.R. Netz, Langmuir 26 (10), 7370 (2010).

[114] D. Argyris, D.R. Cole and A. Striolo, ACS Nano 4 (4), 2035 (2010).

[115] R. O'Brien and L. White, J. Chem. Soc. Faraday Trans. 74, 1607 (1978). 
[116] G. Giupponi and I. Pagonabarraga, Physical Review Letters 106 (24), 248304 (2011).

[117] G. Giupponi and I. Pagonabarraga, Phil. Trans. Roy. Soc. London A 369 (24), 2546 (2011).

[118] B. Dünweg, V. Lobaskin, K. Seethalakshmy-Hariharan and C. Holm, Journal of Physics: Condensed Matter 20 (40), 404214 (2008).

[119] V. Lobaskin, B. Dünweg, M. Medebach, T. Palberg and C. Holm, Phys. Rev. Lett. 98, 176105 (2007).

[120] Y. Nakayama, K. Kim and R. Yamamoto, Europ. Phys. J. E 26, 361 (2008).

[121] O. Hickey, C. Holm, J. Harden and G. Slater, Phys. Rev. Lett. 105, 148301 (2010).

[122] K. Grass and C. Holm, Farad. Disc. 144, 57 (2010).

[123] K.M. Wu, Y.F. Wei and P.Y. Hsiao, Electrophoresis 32 (23), 3348 (2011).

[124] J.F. Dufrêche, B. Rotenberg, V. Marry and P. Turq, Anais da Academia Brasileira de Cincias $82(1), 61(2010)$

[125] V. Marry, B. Rotenberg and P. Turq, Phys. Chem. Chem. Phys. 10 (32), 4802 (2008).

[126] B. Rotenberg, V. Marry, N. Malikova and P. Turq, Journal of Physics: Condensed Matter 22 (28), 284114 (2010).

[127] B. Rotenberg, A.J. Patel and D. Chandler, Journal of the American Chemical Society 133 (50), 20521 (2011).

[128] C.Y. Pealber and S. Baldelli, The Journal of Physical Chemistry Letters 3 (7), 844 (2012).

[129] S. Perkin, T. Albrecht and J. Klein, Physical Chemistry Chemical Physics 12 (6), 1243 (2010).

[130] S. Perkin, Physical Chemistry Chemical Physics 14 (15), 5052 (2012).

[131] A.A. Kornyshev, The Journal of Physical Chemistry B 111 (20), 5545 (2007).

[132] X. Jiang and R. Qiao, The Journal of Physical Chemistry C 116 (1), 1133 (2011).

[133] V. Knecht, H.J. Risselada, A.E. Mark and S.J. Marrink, Journal of colloid and interface science 318 (2), 477 (2008).

[134] S. Joseph and N.R. Aluru, Physical Review Letters 101 (6), 064502 (2008).

[135] D.J. Bonthuis, K. Falk, C.N. Kaplan, D. Horinek, A.N. Berker, L. Bocquet and R.R. Netz, Physical review letters 105 (20), 209401 (2010).

[136] D.J. Bonthuis, D. Horinek, L. Bocquet and R.R. Netz, Langmuir 26 (15), 12614 (2010).

[137] K.F. Rinne, S. Gekle, D.J. Bonthuis and R.R. Netz, Nano Letters 12 (4), 1780 (2012).

[138] A. Siria, P. Poncharal, A.L. Biance, R. Fulcrand, X. Blase, S.T. Purcell and L. Bocquet, Nature 494 (7438), 455 (2013).

[139] D. Seeliger, C. Hartnig and E. Spohr, Electrochimica Acta 50 (21), 4234 (2005).

[140] S. Dokmaisrijan and E. Spohr, Journal of Molecular Liquids 129 (1-2), 92 (2006).

[141] S. Vartak, A. Roudgar, A. Golovnev and M. Eikerling, The Journal of Physical Chemistry B 117 (2), 583 (2013).

[142] F. Wilhelm, W. Schmickler, R.R. Nazmutdinov and E. Spohr, The Journal of Physical Chemistry C 112 (29), 10814 (2008).

[143] F. Wilhelm, W. Schmickler, R. Nazmutdinov and E. Spohr, Electrochimica Acta 56 (28), 10632 (2011).

[144] S.V. Churakov, Geochimica et Cosmochimica Acta 71 (5), 1130 (2007).

[145] C. Zhang, D.G. Knyazev, Y.A. Vereshaga, E. Ippoliti, T.H. Nguyen, P. Carloni and P. Pohl, Proceedings of the National Academy of Sciences 109 (25), 9744 (2012).

[146] CP2K, http://cp2k.org .

[147] VASP, https://www.vasp.at .

[148] LAMMPS, http://lammps.sandia.gov .

[149] GROMACS, http://www.gromacs.org .

[150] ESPResSo, http://espressomd.org .

[151] DL_MESO, http://www.stfc.ac.uk/cse/25522.aspx .

[152] S. Joseph and N.R. Aluru, Langmuir 22 (21), 9041 (2006).

[153] M. Bugel, G. Galliero and J.P. Caltagirone, Microfluidics and nanofluidics 10 (3), 637 (2011).

[154] F. Detcheverry and L. Bocquet, Physical Review Letters 109 (2), 24501 (2012).

[155] N.K. Voulgarakis and J.W. Chu, The Journal of chemical physics 130, 13411 (2009).

[156] B.Z. Shang, N.K. Voulgarakis and J.W. Chu, The Journal of chemical physics 135, 044111 (2011).

[157] B.Z. Shang, N.K. Voulgarakis and J.W. Chu, The Journal of Chemical Physics 137 (4), 044117 (2012).

[158] B. Dünweg, U.D. Schiller and A.J. Ladd, Computer Physics Communications 180 (4), 605 (2009).

[159] S. Thampi, I. Pagoabarraga and R. Adhikari, Phys. Rev. E 84, 046709 (2011).

[160] S.T.T. Ollila, C. Denniston, M. Karttunen and T. Ala-Nissila, The Journal of Chemical Physics 134 (6), 064902 (2011). 